\title{
Heparin Potassium
}

National Cancer Institute

\section{Source}

National Cancer Institute. Heparin Potassium. NCI Thesaurus. Code C113690.

The potassium salt form of heparin, a sulfur-rich glycosaminoglycan with anticoagulant activity. Heparin binds to antithrombin III to form a heparin-antithrombin III complex. The complex binds to and inactivates thrombin (factor Ila) and other activated clotting factors, such as factors IXa, Xa, Xla, and XIIa. The inactivation of thrombin prevents the polymerization of fibrinogen to fibrin and the subsequent formation of blood clots. 